\title{
超分子有机框架：水相周期性自组装孔结构
}

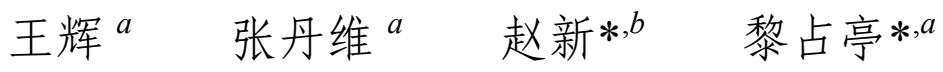 \\ $\left({ }^{a}\right.$ 复旦大学化学系 上海 200433) \\ ( ${ }^{b}$ 中国科学院上海有机化学研究所 有机功能分子合成与组装化学重点实验室 上海 200032)
}

\begin{abstract}
摘要 概述了水相超分子有机框架组装方面最近的进展. 在简述了利用多头基单体构筑超分子组装体的背景之后，介 绍了利用三头基和四头基分子形成超分子网络组装体的重要例子，着重描述了利用刚性预组织平面三角形单体和四面 体单体，通过葫芦嫝[8]对 4-苯基吡啶盐堆积二聚体的包结作用，在水相构筑二维和三维超分子有机框架的进展，并简 要叙述了在水相表征周期性自组装结构的仪器分析方法. 最后就超分子有机框架构筑的单体设计及其可能的应用及功 能做了分析与展望.
\end{abstract}

关键词 超分子有机框架; 孔结构; 周期性; 疏水作用; 水相

\section{Supramolecular Organic Frameworks (SOFs): Water-Phase Periodic Porous Self-Assembled Architectures}

\author{
Wang, Hui ${ }^{a} \quad$ Zhang, Dan-Wei ${ }^{a} \quad$ Zhao, Xin ${ }^{*}, b \quad$ Li, Zhan-Ting*, \\ ( ${ }^{a}$ Department of Chemistry, Fudan University, Shanghai 200433) \\ ( ${ }^{b}$ Key Laboratory of Synthetic and Self-Assembly Chemistry for Organic Functional Molecules, Shanghai Institute of \\ Organic Chemistry, Shanghai 200032)
}

\begin{abstract}
This article summarizes the recent advance for the construction of supramolecular organic frameworks (SOFs) in aqueous media from rationally designed rigid preorganized building blocks. We first introduce the research background on the design of multitopic molecular monomers for the self-assembly of discrete supramolecular aggregates and polymers. We then describe the formation of less ordered supramolecular polymers from tritopic molecular monomers. In the following section, we show that conjugated rigid triangular building blocks have been successfully applied for the construction of two-dimensional (2D) one-layer SOFs in water. Following this, we further present the design of tetratopic building blocks for the formation of three-dimensional (3D) supramolecular networks in aqueous and organic solvents. Finally, we demonstrate that a 3D porous SOF can be assembled from a preorganized tetrahedral building block in water. For the formation of both 2D and 3D SOFs, the hydrophobically driven encapsulation of the stacked dimer of 4-phenylpyridinium unit in the cavity of cucurbit[8]uril in water plays a key role, and the dimerization of viologen radical cation has also been utilized as driving force for generating a 2D SOF. We also briefly introduce the analytical methods for the characterization of SOFs in solution. In the conclusion section, we make a perspective for the construction of SOFs in solution and their potential applications in guest adsorption and release.

Keywords supramolecular organic framework; porous structure; periodicity; hydrophobicity; aqueous solution
\end{abstract}

\section{1 引言}

分子自组装利用可逆非共价键相互作用力把合理设 计的分子单体 “拼装” 成不同形状的分子集合体 ${ }^{[1 \sim 8]}$. 如 果单体间的作用力具有方向性, 相应的组装体将会形成 特定的形状. 把两个及更多的作用力引入到一个单体中, 可以产生相应的双头基或多头基单体. 理论上，当这些 作用力都具有方向性，而单体骨架又具有刚性时，其可 以在二维和三维空间自组装形成有序性的阵列结构 ${ }^{[9]}$. 这一过程类似于有机分子在晶体中的周期性堆积. 但分 子在晶体中的堆积不受溶剂的影响. 因此, 即使在分子
间的相互作用力很弱时，分子仍可能形成周期性的排列. 但在溶液中, 分子具有固有的发生扩散及布朗运动的倾 向性，通过定向的分子间相互作用力把单体分子组合在 一起, 形成有序的周期性结构, 从熵变的角度看是一个 非常不利的过程. 因此，单体间的结合首先必须强度高， 产生大的结合焓, 才能把很多的单体分子拉近并结合在 一起. 另外，单体分子间的相互作用力还必须具有方向 性, 从而使相互结合的单体在空间上有序排列, 进而形 成周期性的组装结构. 在过去的 50 多年中，尽管分子识 别、超分子化学、分子自组装及超分子晶体工程领域的 研究进展迅速 ${ }^{[10 ~ 21]}$, 在 2013 年之前, 组装溶液相周期性

\footnotetext{
* E-mail: ztli@fudan.edu.cn; Tel.: 021-65642038; Fax: 021-65641740

Received December 22, 2014; published February 4, 2015.

Project supported by the National Natural Science Foundation of China (No. 91227108).

项目受国家自然科学基金(No. 91227108)资助.
} 
的超分子框架阵列结构，国内外从未有报道.

基于结构的周期性，物理学家发展了 $\mathrm{X}$ 射线衍射技 术, 用于测定分子和大分子的精确结构. 周期性也是很 多晶体材料展示光、电、磁等性能的结构基础. 就分子 晶体、配合物晶体及配位聚合物晶体而言, 当结合位点 间的距离较大，而连接结合位点的分子骨架刚性较高 时, 整个结构将形成孔型框架, 从而能包埋溶剂分子或 不同的客体分子. 当空穴较大时, 可以在保持晶体框架 结构的情况下发生客体交换 ${ }^{[22 ~ 24]}$. 在过去的 20 多年内, 有关金属有机框架(Metal-Organic Framework, MOF)的 研究是无机和材料化学研究的最活跃领域之一 $[25 \sim 30]$. 周期性和孔穴也是 MOF 材料的两个最重要结构特征, 不但赋予其大的比表面积, 还提供了吸附、吸收、释放、 运载、识别、传感及催化等不同方面的功能及应用潜力. 近 10 年来发展的共价有机框架 (Covalent-Organic Framework, COF)也具有类似的周期性和空穴特征, 其不 同之处在于框架的形成是通过可逆共价键实现的 ${ }^{[31 ~ 34]}$.

无论是晶体、MOF 或 COF 结构, 它们都是典型的固 体 “硬” 材料, 很难在溶液相稳定存在或不具备溶解性. 目前, 化学家和物理学家也可以通过各种技术在界面或 表面构造不同的周期性网络结构 ${ }^{[35,36]}$. 但这些有序结构 目前还不能实现剥离, 或转移到溶液相中. 因此, 发展新 的手段, 在溶液相构筑周期性的框架或孔结构, 将为揭 示新的科学现象和规律及发展新的材料提供物质基础, 无论从基础和应用两方面来看, 都是非常重要的.

过去的 20 多年见证了分子自组装技术的快速发展. 目前, 超分子化学家已经设计了大量的多头基单体, 用 于构筑各种各样的超分子聚合物网络结构 ${ }^{[37,38]}$. 许多超 分子网络结构展示出独特的性质或性能, 如控制释放、 催化及感应等. 形成超分子网络结构的单体可以是双头 基、三头基或四头基型的有机分子, 也可以是侧链结合 型的大分子等. 这些单体分子和大分子一般由柔性的或 非全刚性的骨架形成, 形成的超分子网络结构虽然具有 三维的网络结构, 但在二维及三维空间一般都不具备有 序性或周期性. 从自组装的角度来看, 如果单体间的结 合作用力足够强并具有方向性, 理论上应可以在溶液相 形成周期性的框架结构. 这种溶液相的有序结构不但本 身结构独特, 而且也预期具备很多的应用潜力. 比如, 目前发展的晶体及 MOF 和 COF 材料与孔结构相关的许 多性质和功能, 在溶液相都有可能通过自组装框架结构 加以实现. 另外, 均相的自组装框架作为一类新的 “软” 结构还可能展示出新的独特性质, 比如有驱动力的主动 吸附、客体吸附的定量化、客体吸附的快速实现及热力 学和动力学控制、更高的可修饰性和刺激响应性等.

理论上, 在溶液中, 周期性的自组装结构可以在二 维和三维空间构筑. 就二维结构来说, 对称的三角、四 角及六角形的三、四及六头基单体可以形成单一周期的 平面网格结构. 而单一周期的三维结构可以通过四面体
及八面体的四头基和六头基单体组装而成. 我们目前已 经在二维空间实现了从三角形分子单体构筑蜂窝型网 格结构，在三维空间实现了从四面体分子单体构筑类金 刚石型孔结构，建立了有序结构表征的方法，并初步探 索了后者的吸收和释放功能. 本文将首先介绍基于三头 基和四头基单体的超分子聚合物组装, 然后介绍我们在 超分子有机框架组装方面的探索. 我们把这类溶液相的 周期性自组装结构称为超分子有机框架(Supramolecular Organic Framework, SOF), 以表示其基于有机分子形成 的超分子和有序孔结构特征. 事实上, $\mathrm{S}$ 也可以代表自 组 装 (Self-assembled)、可溶性 (Soluble) 或 溶液相 (Solution)等含义, 因为周期性、均相、可溶性及有空穴 是这类自组装结构的最显著特征.

\section{2 三头基单体构筑超分子聚合物}

三头基分子被广泛应用于各种分子和大分子体系 的构筑中 ${ }^{[39,40]}$. 从拓扑学上讲, 三头基分子也适合于构 筑网络结构. 早期 Whitesides 等 ${ }^{[1]}$ 即是利用三聚氰胺和 三聚氰酸形成二维氢键蜂窝型网络结构, 以说明分子自 组装的概念. 这类自组装结构可以在晶体或界面形成.

Lehn 等 ${ }^{[41]}$ 报道，并入三个 2,6 -二酰基吡啶的三头基 单体 1 和并入两个三聚㲵酸的双头基单体 2 通过形成稳 定的互补 ADA-DAD ( $\mathrm{A}=$ acceptor, $\mathrm{D}=$ donor) 双三氢键

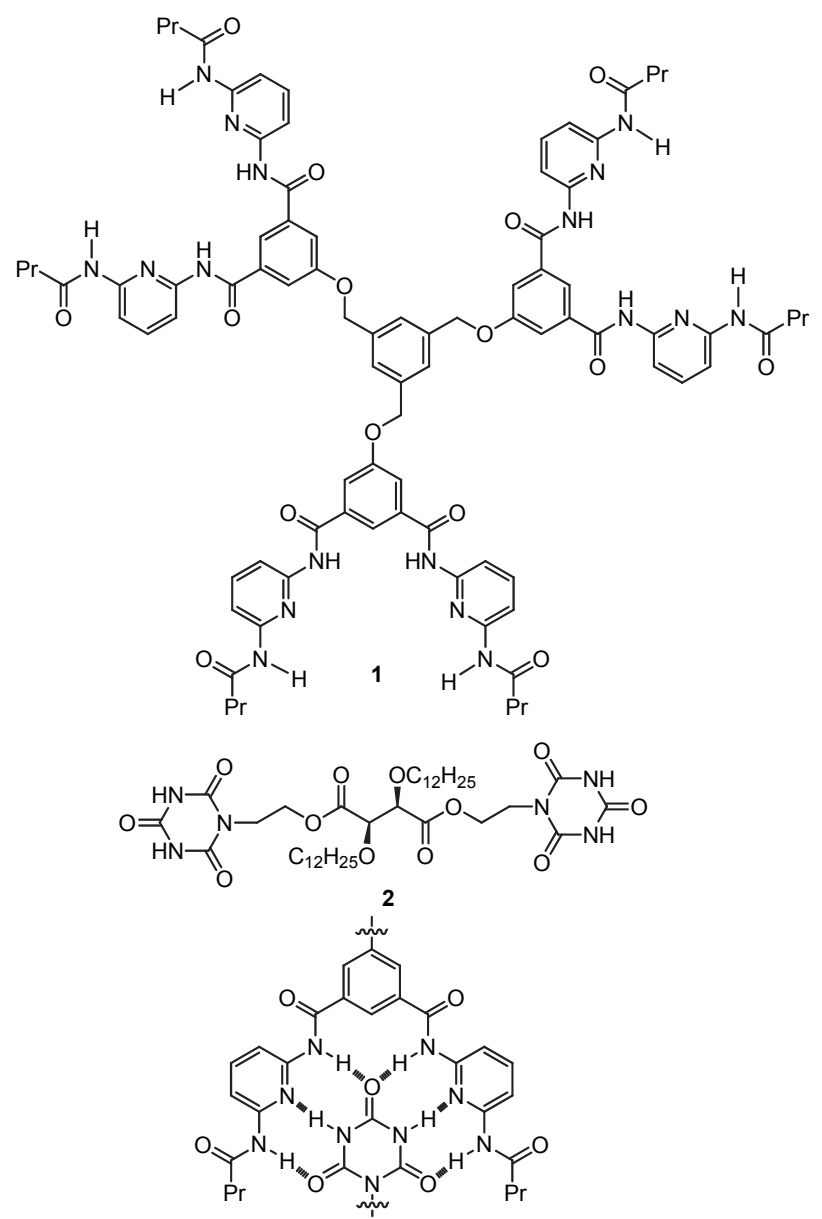


二聚体, 可以形成超分子聚集体团簇结构, 而等物质量 的并入双头基的 2,6-二酰基吡啶片段的分子可以与 2 结 合, 则形成纤维结构. 这些结果说明, 三头基的单体 $\mathbf{1}$ 可以在二维和三维空间形成网络型结构, 而双头基分子 则只能形成线性的超分子聚合物.

Lightfoot 等 ${ }^{[42]}$ 报道 1,3,5-间苯三甲酰胺衍生物 3a $3 \mathrm{e}$ 等形成分子间氢键及芳环堆积. 在晶体中这类三头 基分子不形成二维的自组装结构, 而是形成一维柱状堆 积体. 对于共轭三角型分子, 中间的大共轭骨架的堆积 作用决定了分子的堆积模式, 侧链上的氢键进一步强化 了这一堆积作用. 当侧链上引入手性中心时(3e), 相应 的一维堆积结构可以形成手性螺旋纤维 ${ }^{[43]}$. 在液相中 可以通过圆二色谱验证, 在固态, 这种纤维结构可以通 过高分辨率 AFM 或 TEM 等观察.<smiles>[R]CCCC(C)CCCC(C)C</smiles>

Zhang 等 ${ }^{[44]}$ 合成了水溶性的三头基分子 4, 在外围 引入三个菜环, 其外围的菜环具有疏水性, 在水中可以 与葫芦脲 $(\mathrm{CB}[8])$ 通过疏水作用形成 $2: 1$ 络合物. 可能 是由于三氮唑正离子间的排斥作用, 两个萗环选择性反 方向堆积形成二聚体, 被包结于 $\mathrm{CB}$ [8]的疏水空腔内. 由于这一包结作用具有很高的稳定性. 因此, 二者物质 的量之比为 $2: 3$ 的混合物可以在水中形成超支化型的 超分子聚合物(图 1).

Tian 和 $\mathrm{Ma}$ 等 ${ }^{[45]}$ 合成了水溶性紫精三头基单体 $\mathbf{5}$, 在外围引入三个香豆素片段. 受疏水作用驱动, 两个香 豆素可以被 $\gamma$-环糊精包结, 形成 $2: 1$ 络合物, 形成网络 型超分子聚合物 NNP(图 2). 在 $254 \mathrm{~nm}$ 紫外光照射下, 香豆素发生光二聚反应, 形成共价键, 从而把超分子聚 合物转变为共价聚合物 CNP. 在 $365 \mathrm{~nm}$ 紫外光照射下, 二聚体可以解聚, 从而恢复到超分子聚合物 NNP, 整个 体系构成一个可以可逆转换的自组装体系.

$\mathrm{Li}$ 和 $\mathrm{Jia}$ 等 ${ }^{[46]}$ 设计合成了三头基分子 6 , 其外侧的 三氮唑氰基脂肪链可以在氯仿中被柱[5]芳烃包结. 在 氯仿中, 物质的量之比为 $2: 3$ 的 6 和双柱[5]芳烃 7 混 合物可以形成另一类超支化型的超分子聚合物. 在高浓 度下其增比粘度明显提高并形成纤维结构.

Haino 等 ${ }^{[47]}$ 合成了刚性的三头基分子 8 , 在其外侧 引入三个吡啶相连的双杯 [5]芳烃单元. 在氯仿或甲苯 中, 这一双杯 [5]芳烃单元可以与 $\mathrm{C}_{60}$ 形成稳定的络合物. 因此, 它与并入两个 $\mathrm{C}_{60}$ 单元的双头基分子 9 结合, 在有

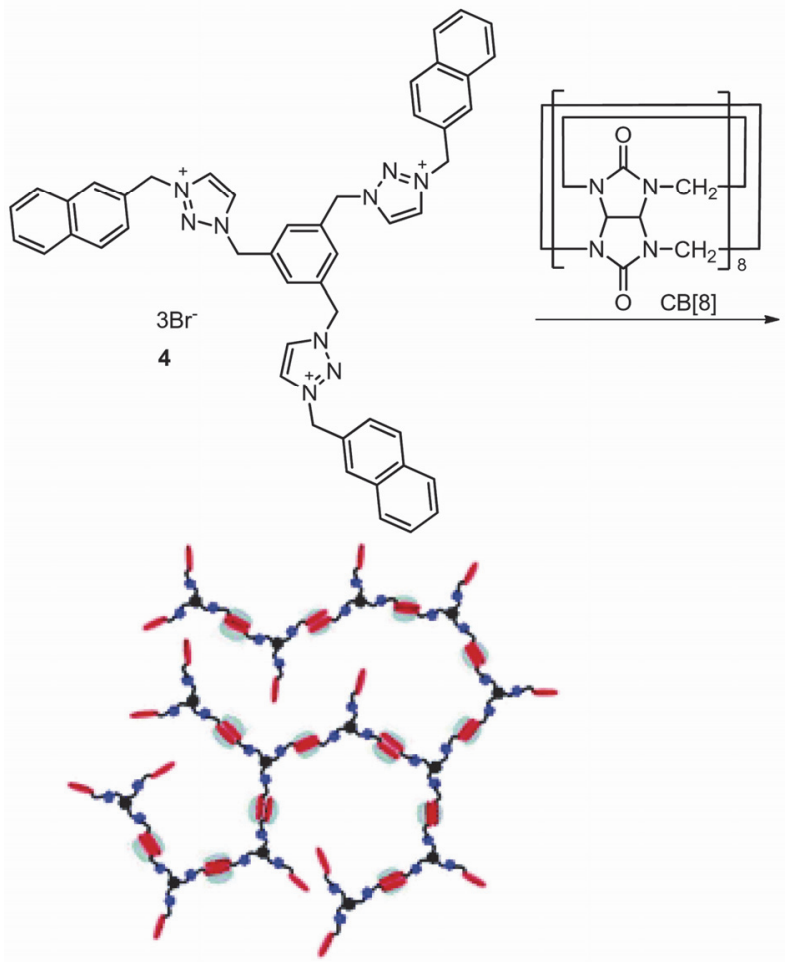

图 1 疏水作用驱动 $\mathrm{CB}[8]$ 包结化合物 $\mathbf{4}$ 的萗环堆积二聚体形成超支 化超分子聚合物 ${ }^{[44]}$

Figure 1 Schematic representation of the formation of a supramolecular hyperbranched polymer by hydrophobically driven encapsulation of the naphthalene stacking dimer of compound 4 by $\mathrm{CB}[8]^{[44]}$

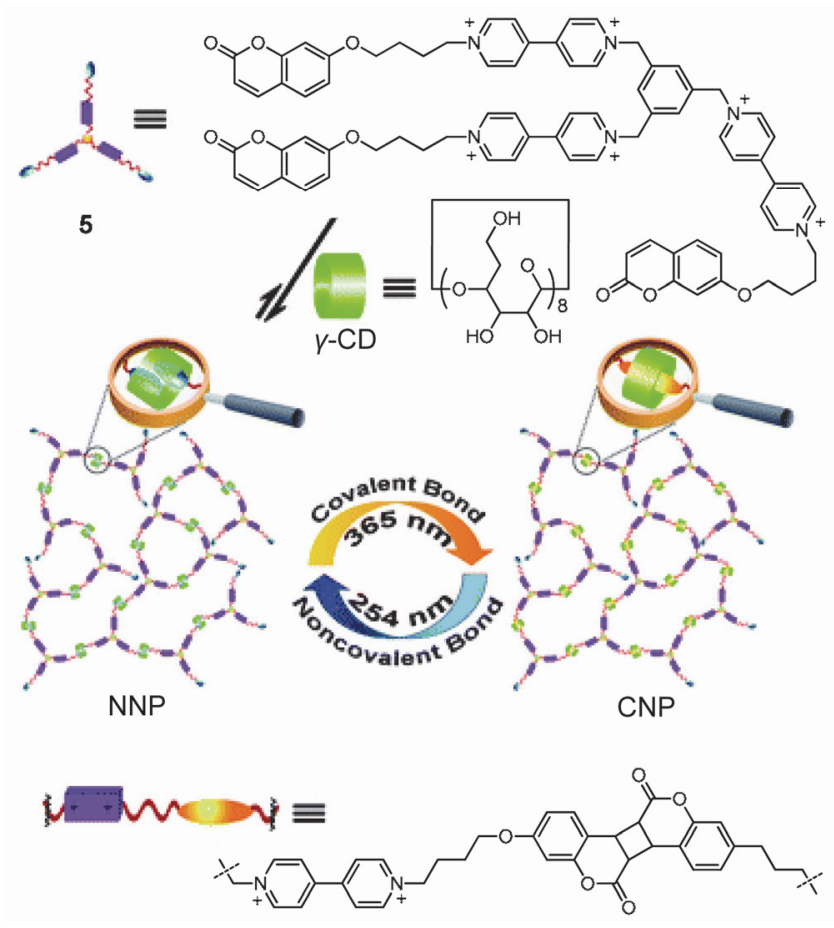

图 2 三头基单体 $\mathbf{5}$ 和 $\gamma$-环糊精受疏水作用驱动形成网格型超分子聚 合物(NNP)

Figure 2 Schematic representation for the formation of supramolecular polymer NNP by host-guest interaction between tritopic monomer $\mathbf{5}$ and $\gamma$-CD

The photo-switching between NNP and its corresponding covalent polymer CNP by alternating UV light irradiation at 254 and $365 \mathrm{~nm}$, respectively ${ }^{[45]}$ 

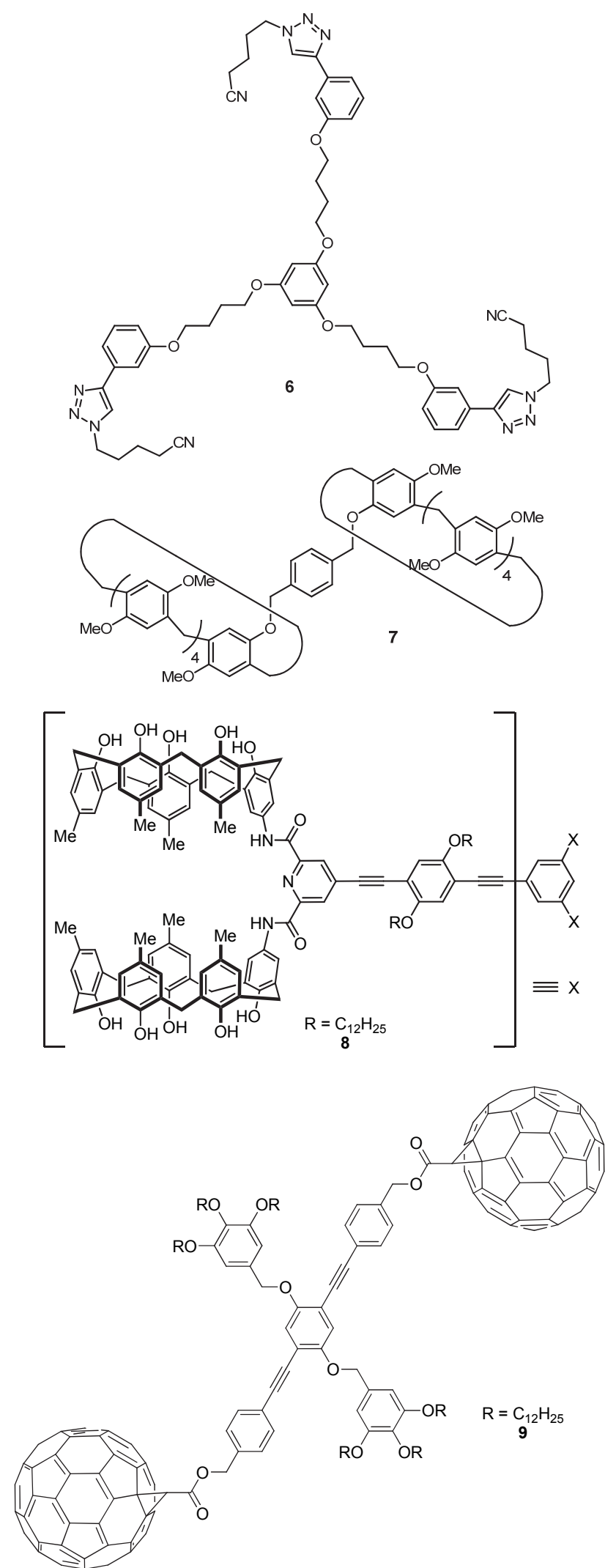

机溶剂中可以形成超分子网络体系. SEM 显示, 这一组 装体系在表面产生一些不规则的孔结构, 表明三角形的 刚性骨架具有一定的结合方向性, 可能有利于形成二维 的自组装体系.

\section{3 刚性共轭三头基单体构筑单层超分子有机框} 架

上述三头基分子都不具备完整的分子骨架刚性. 我 们推测，通过增强单体的刚性提高分子间作用力的方向 性是实现二维周期性框架结构组装的关键. 为此, 我们 设计合成了一个全刚性的 1,3,5-三苯基衍生物 $\mathbf{1 0}^{[48]}$, 在 其外围引入三个 $4,4^{\prime}$-双吡啶单正离子(BP)单元, 并在中 心的苯环上引入三个亲水的 $N, N$-二羟乙基酰胺片段. 这 三个亲水链一方面抑制三角型骨架的一维堆积, 另一方 面能提供水相中的溶解度. BP 单元在水中可以与葫芦 腿[8](CB[8])形成 $2: 1$ 的包结络合物 ${ }^{[9,50]}$. 把 $\mathbf{1 0}$ 和 $\mathrm{CB}[8]$ 以 $2: 3$ 的物质的量之比在水中混合, 可以形成单 层的二维 SOF 结构, 驱动力来自于疏水作用驱动的 $\mathrm{CB}[8]$ 对其外侧 $\mathrm{BP}$ 堆积二聚体的包结作用.<smiles></smiles>

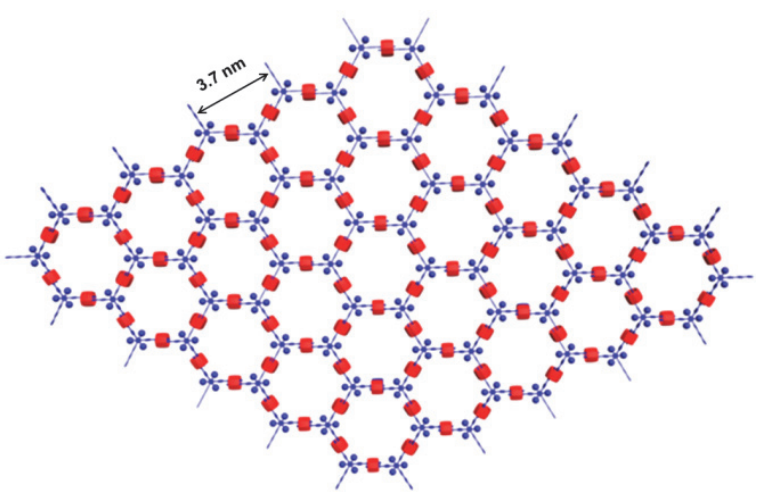

图 3 刚性平面三头基分子 $\mathbf{1 0}$ 和 $\mathrm{CB}[8]$ 形成的二维 $\mathrm{SOF}$ 结构 ${ }^{[48]}$ Figure 32 D periodic SOF formed by rigid planar triangular molecule 10 and $\mathrm{CB}[8]^{[48]}$

这一 SOF 结构的周期性得到 ${ }^{1} \mathrm{H}$ 核磁、动态光散射、 $X$ 射线散射和衍射、SEM、TEM 和 AFM 等实验验证. 溶 液相同步辐射 $X$ 射线散射图显示一个对应于 $3.61 \mathrm{~nm}$ 间 距的散射峰，与蜂窝型二维结构的六方形单元平行线间 距的计算值 $(3.70 \mathrm{~nm})$ 非常吻合. 溶液相同步辐射 X 射线 衍射图也显示一个对应于 $3.78 \mathrm{~nm}$ 间距的衍射峰. AFM 图揭示这一组装体的高度平均约为 $1.72 \mathrm{~nm}$, 与 $\mathrm{CB}[8]$ 的 
外直径 $(1.75 \mathrm{~nm})$ 非常吻合, 从而证实了组装体的单层结 构特征. 提高浓度组装体可以形成水凝胶, 其固体 $\mathrm{X}$ 射 线衍射实验表明, 组装体在固态保持其周期性有序结构.

化合物 10 可以进一步被甲基化形成刚性三紫精单 体 ${ }^{[51]}$. 这一水溶性分子在水中可以被锌粉还原为紫精 正离子自由基衍生物 11. 由于整个分子骨架的全刚性 共平面特征, 其外侧的三个紫精正离子自由基可以发生 分子间的二聚, 在二维空间形成蜂窝型 SOF 组装结构, 其周期性得到小角 X 射线衍射实验证实. 紫精正离子自 由基二聚体可以被 $\mathrm{CB}[8]$ 包结 ${ }^{[52]}$. 因此, 加入 $\mathrm{CB}[8]$ 能 进一步提高这一二维 SOF 的稳定性及有序性.

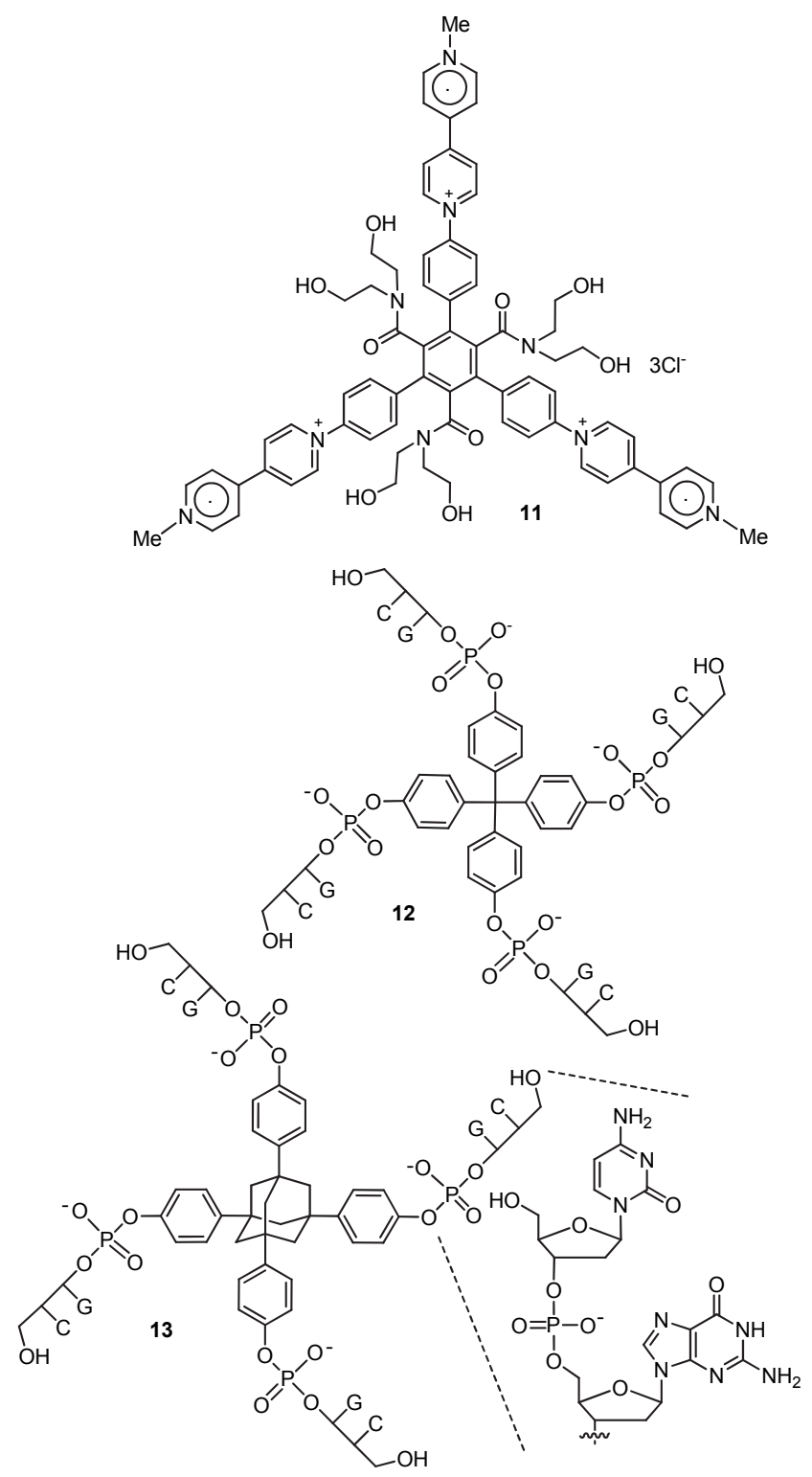

\section{4 四头基单体构筑超分子网络结构}

四面体的四头基单体可以在三维空间形成分子间 结合. Richert 等 ${ }^{[53]}$ 合成了四苯甲烷和金刚烷衍生物 12 和 13 , 其外侧的核苷链可以通过两个 $\mathrm{G} \cdot \mathrm{C}$ 碱基对氢键
形成分子间结合．简单的 $\mathrm{G} \cdot \mathrm{C}$ 碱基对由于太短在水中不 能形成稳定的氢键, 但并入到 12 和 13 骨架的 GC 核苷 链可以形成稳定的二聚体结构，从而诱导产生超分子聚 合物网络结构. 这一分子间氢键结合强度相当高，因此 在 $95^{\circ}$ 时两个分子就可以形成沉淀. 两个分子的刚性四 面体骨架应是增强其分子间相互作用的一个关键因素.

Wang 和 Zhang 等 ${ }^{[54]}$ 也合成了卟啉衍生物 14, 在卟 啉的四个 meso 位引入四个疏水萗环. 在水中受疏水作 用驱动, 不同分子的两个菜环可以被包结在 $\mathrm{CB}[8]$ 中, 这一 2: 1 的络合模式诱导 14 形成超支化超分子聚合物. 尽管卟啉骨架是平面刚性的，由于亚甲基的引入，周围 的䒺环可以偏离卟啉平面旋转, 因此, 整个组装结构在 三维空间形成, 而不局限在卟啉决定的二维平面内.

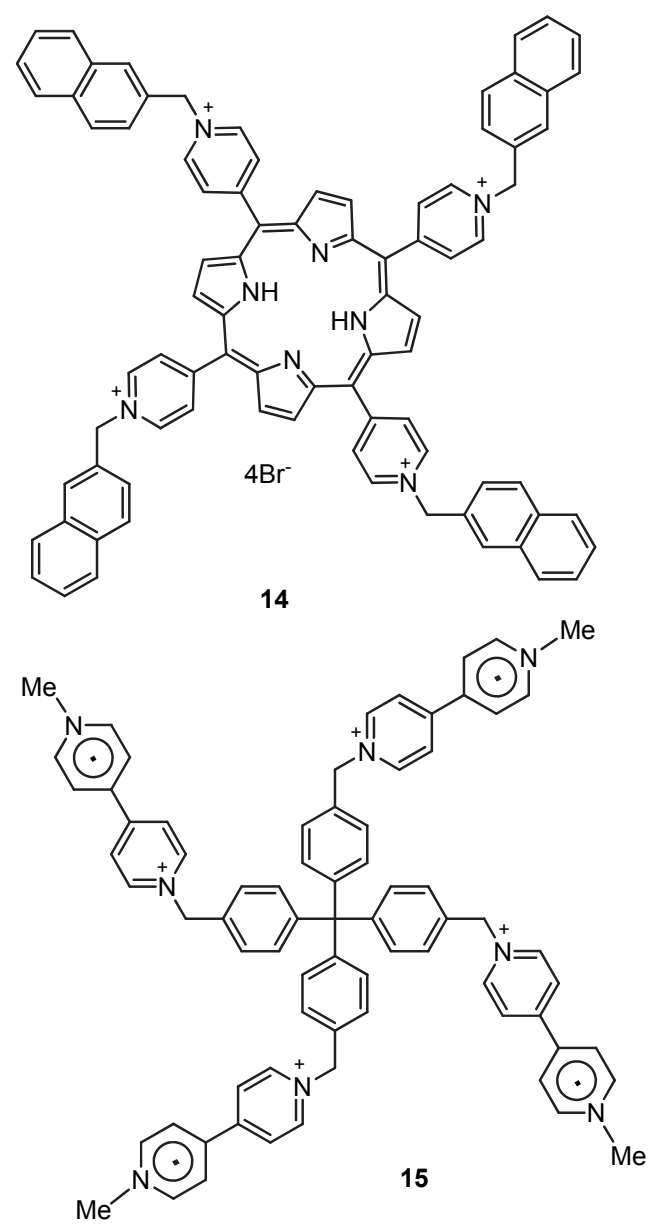

我们研究组基于四苯基甲烷骨架设计合成了并入 四个紫精片段的单体 ${ }^{[55]}$. 在水中紫精片段可以被保险 粉还原为正离子自由基. 相应的单体 15 的紫精正离子 自由基发生分子间堆积或二聚，从而驱动形成超分子网 络结构. 紫精正离子自由基二聚的结合常数与控制化合 物比较高出很多, 意味着四面体结构提高了四个结合单 元的二聚协同性. 超分子聚合物的形成得到紫外-可见 吸收光谱、电化学、电子顺磁共振实验的验证. 动态光 散射实验表明, 这一超分子聚合物在水相中的动态力学 直径大约为 $34 \mathrm{~nm}$. 这一尺寸相当于单个化合物直径(大 
约 $3.3 \mathrm{~nm}$ )的 11 倍, 意味着一个组装体由上千个单体分 子组成.

我们还设计合成了四苯基甲烷衍生物 $16 \mathrm{a}$ 和 $16 \mathrm{~b}$, 它们分别带有四个四硫富瓦烯(TTF)单元 ${ }^{[56]}$, 其 TTF 单 元可以同时被高氯酸铁等氧化剂氧化为正离子自由基 $\mathrm{TTF}^{{ }^{+}}$. 这些 $\mathrm{TTF}^{*+}$ 单元受结构预组织构象的驱动, 在有 机溶剂中发生强的分子间堆积或二聚, 诱导形成三维的 超分子聚合物. $16 \mathrm{~b}$ 的正离子自由基衍生物在水中也具 有一定的溶解性, 其在水中也可以发生簇集形成三维超 分子网络结构. 动态光散射实验表明, 在乙腈水溶液 $(1: 1)$ 中, 16a 和 $\mathbf{1 6} \mathbf{b}$ 可以形成平均流体力学半径为 34 和 $53 \mathrm{~nm}$ 的超分子聚集体, SEM 和 AMF 揭示聚集体为 球形结构. 在有机溶剂中, $16 \mathrm{c}$ 和 $16 \mathrm{~d}$ 的正离子自由基衍 生物也可以形成类似的聚集体 ${ }^{[57]}$.
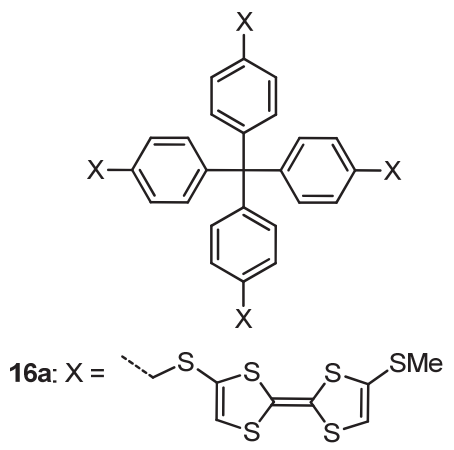<smiles>COCC=[GeH2]</smiles>

16c: $X=$<smiles>CNC(=O)C1=CSC(=C2SC=CS2)S1</smiles>

16d: $X=$<smiles>CC#CC1=CSC(=C2SC=CS2)S1</smiles>

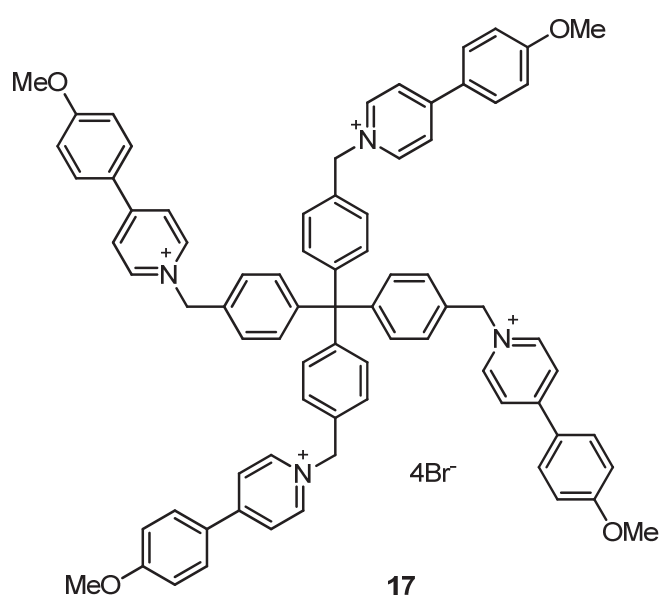

\section{5 通过互锁结合模式构筑水相三维超分子有机}

\section{框架}

上述四面体单体通过分子间氢键及疏溶剂作用驱
动形成的三维超分子结构的稳定性是相当高的，在高浓 度下可以形成粒径在 $100 \mathrm{~nm}$ 左右的聚集体. 但所有这 些超分子组装体都没有证据表明，其形成了周期性的有 序结构.一个可能的原因是, 这些聚集体的结合位点在 溶液中处于动态, 会发生摆动、旋转、伸缩甚至于解离 等, 从而降低了单体在空间排列的周期性. 利用大环化 合物络合锁住结合位点，一方面可以进一步提高识别位 点间的结合稳定性，另一方面也可以降低上述可能的分 子运动. 基于以上考虑，我们合成了四面体单体 $17^{[58]}$, 在其外侧引入四个疏水的 4-苯基吡啶正离子, 通过葫芦 嫝[8]对该芳香片段的 $1: 2$ 包结作用，在水相中构筑了 一类空穴型的三维 SOF 结构(图 4).

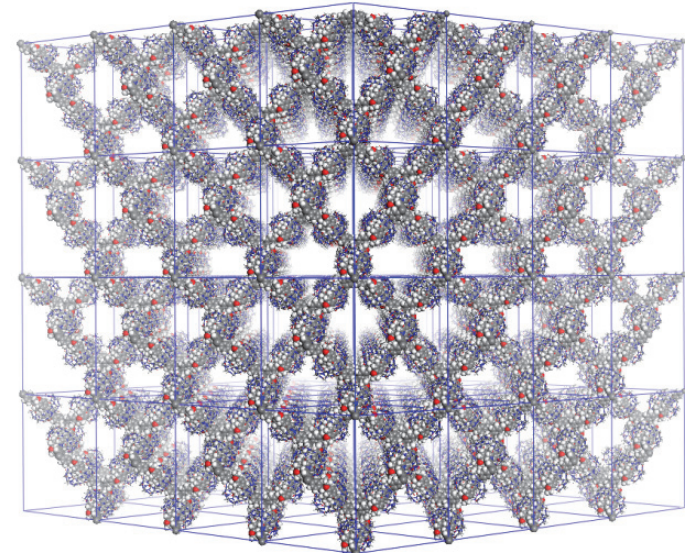

图 4 模拟的由四头基分子 $\mathbf{1 7}$ 和 $\mathrm{CB}[8]$ 形成的三维超分子有机框架结 构 ${ }^{[58]}$

Figure 4 3D periodic SOF formed by tetrahedral molecule 17 and $\mathrm{CB}[8]^{[58]}$

这一三维组装体的周期性结构特征在水相得到 X 射线散射和衍射实验验证(图 5). 这类三维 SOF 具有 2.2 $\mathrm{nm}$ 的孔径, 作为一类新的空穴型正离子自组装聚电解 质，可以在水相高效吸收负离子型的染料、药物、肽、 核酸及树枝状分子等. 这类 SOF 结构在固态慢慢结晶, 形成微晶体, 并保持其有序结构, 其周期性特征在固相 也得到小角 $X$ 射线衍射和散射实验证实(图 5). 微晶体 高度稳定, 不溶于水, 可以从水相直接吸收上述客体, 并通过调节 $\mathrm{pH}$ 值实现客体(药物)释放. 这类新型的自 组装框架结构不含过渡金属离子, 细胞毒性很低, 其在 液相和固相的应用潜力值得进一步探索.

\section{6 总结与展望}

综述了近年来基于多头基单体的超分子聚集体的 组装. 柔性或部分柔性的单体形成的组装体易于形成纤 维和无规则的网络结构, 代表了较高层次的自组装结 构，单体间的结合在很多情况下显示出协同性，但方向 性不高, 作为一种无规则的自组装结构与主链和侧链超 分子聚合物相比, 在功能开发上的优势还有待进一步的 研究. 

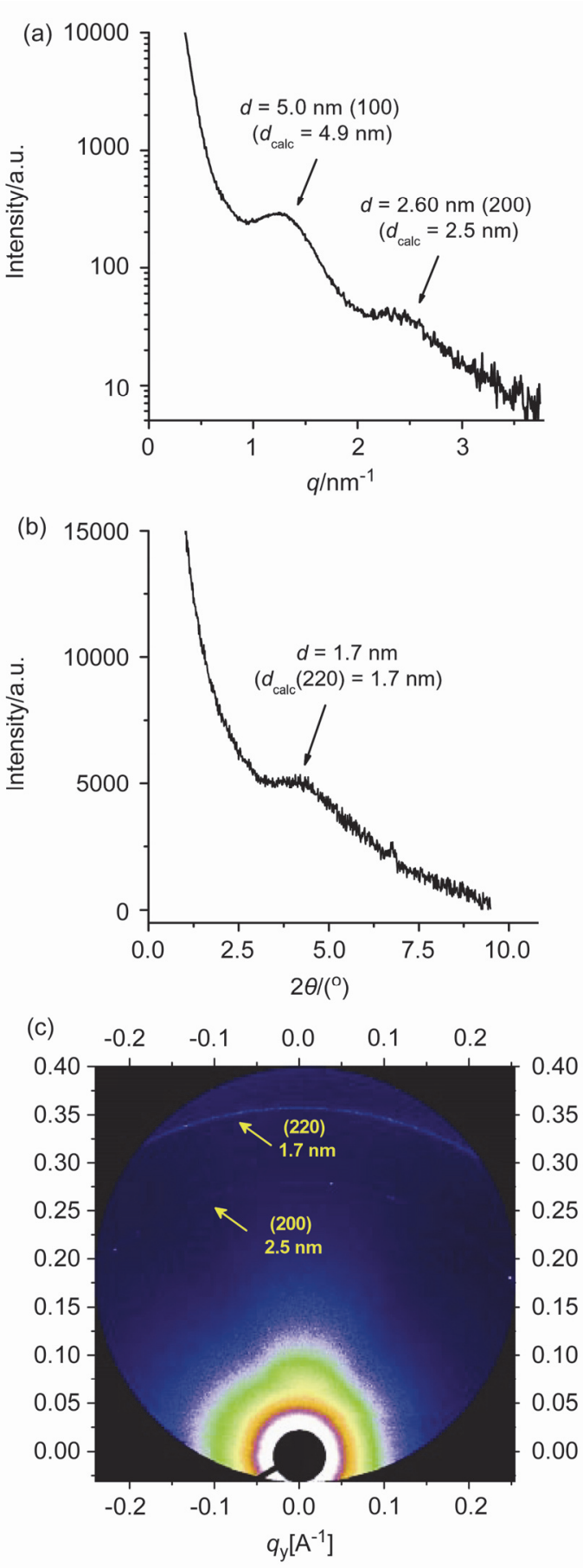

图 5 (a) 水相三维 SOF 同步辐射小角 X 射线散射图(葫芦腿浓度为 4 $\mathrm{mmol} \cdot \mathrm{L}^{-1}$ )、(b) 水相三维 SOF 同步辐射 $\mathrm{X}$ 射线衍射图(葫芦腿浓度为 $4 \mathrm{mmol} \cdot \mathrm{L}^{-1}$ )和(c) 三维 $\mathrm{SOF}$ 固相二维 $\mathrm{X}$ 射线散射图 ${ }^{[58]}$

Figure 5 (a) Synchrotron small-angle X-ray scattering profile of the solution of $3 \mathrm{D}$ SOF in water $\left([\mathrm{CB}[8]]=4 \mathrm{mmol} \cdot \mathrm{L}^{-1}\right)$, (b) Synchrotron $\mathrm{X}$-ray diffraction profile of the solution $3 \mathrm{D}$ SOF in water $([\mathrm{CB}[8]]=4$ $\mathrm{mmol} \cdot \mathrm{L}^{-1}$ ) and (c) $2 \mathrm{D}$ synchrotron X-ray scattering profile of Com-Tetra of the solid sample ${ }^{[58]}$.

我们在多头基单体自组装研究基础上，提出利用单 体骨架的刚性提高单体间结合的强度和方向性, 在水相 成功构筑了二维和三维超分子有机框架结构. 这类自组 装结构的最显著特征是其在溶液相展示出周期性的有
序结构. 尽管共轭自由基正离子堆积二聚已经在水相成 功应用于构筑二维超分子有机框架，利用大环分子对结 合位点的包结更能促进高稳定和高有序性的框架结构 的形成. 我们认为, 就液相分子自组装而言, 超分子有 机框架代表了组装体复杂性和多层次性研究的一个新 的高度. 与通过配位键或离子键构筑的金属有机框架及 通过共价键构筑的共价有机框架相比，超分子有机框架 代表了一类新的溶液相存在的自组装型的 “软” 结构材 料. 拥有纳米尺度的微孔是这类自组装结构的另一个重 要特征. 由于构筑单体都是离子型的, 超分子有机框架 可以认为是一类有序性的超分子聚电解质，其水溶性特 征预示着其在离子型客体的吸附和释放研究方面具有 很大的应用潜力. 自组装超分子有机框架不含有过渡金 属离子, 因此不存在金属有机框架中过渡金属离子带来 的潜在毒性, 这将有利于超分子有机框架在药物输送及 生物材料研究中的应用. 目前, 二维超分子有机框架的 研究还处于开始阶段, 其功能研究还没有报导. 一个潜 在的应用是可作为基底有序排列具有生物或材料性能 的带有相反电荷的分子或大分子. 三维的微晶超分子有 机框架已经展示了独特的负离子吸附吸收和释放功能. 由于吸附吸收的驱动力为较强的静电作用, 这类新的微 晶自组装材料的一个潜在应用是去除水相中的极低浓 度的负离子有机染料及金属氧化物负离子等污染物.

溶液相超分子有机框架概念的建立为自组装研究 提供了新的结构平台. 目前报导的单体都是正离子型 的，我们有理由期望负离子型及中性的组装单体在未来 也可以被设计出来. 框架结构的孔径、新的结合模式的 建立、单体的修饰及功能化等都是值得进一步探索的课 题. 就功能探索来说, 三维结构由于具有孔道更值得关 注. 我们研究的第一个重点是利用三维超分子框架结构 的正电性特征, 吸附负离子型的药物, 通过自组装结构 的水溶性, 发展新的生物靶向试剂及输送体系. 三维超 分子框架还存在一个独特的形成水凝胶的浓度区间. 由 于这类组装结构以互穿形式存在, 系统地对单体进行修 饰, 将有可能发展出新的高度稳定的自修复材料.

致谢 感谢上海市科委、科技部、教育部和国家自然科 学基金委对本课题的资助.

\section{作者简介}

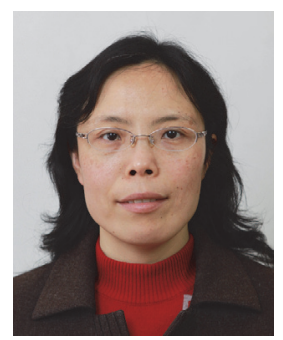

王辉, 1974 年出生于河南, 1996 年毕业于北京科技大学化 学系, 2003 年在北海道大学获得博士学位(导师: 高桥保教授), 
随后在东京工业大学从事博士后研究. 自 2005 年起在复旦大 学化学系工作. 现从事物理有机和超分子化学研究.

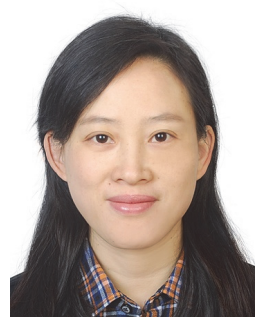

张丹维, 1972 年出生于上海, 1994 年于复旦大学化学系保 送研究生, 2000 年获理学博士学位(导师: 吴世晖教授)后留校 任教. 2001 年在香港大学化学系做访问学者, 2005 年 5 月起为 复旦大学副教授. 现主要从事物理有机和超分子化学研究.

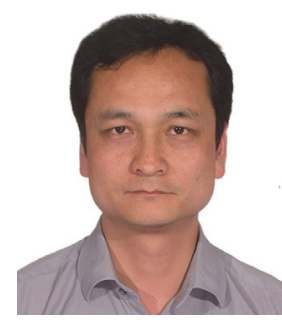

赵新, 1972 年出生于云南, 1994 年毕业于北京师范大学化 学系, 2003 年在中科院上海有机化学研究所获理学博士学位 (导师: 蒋锡熟研究员、黎占亭研究员), 随后在美国哈佛大学 和芝加哥大学从事博士后研究, 自 2008 年 5 月起在中科院上 海有机化学研究所工作, 历任副研究员、研究员. 主要从事超 分子化学、物理有机化学和有机多孔材料研究.

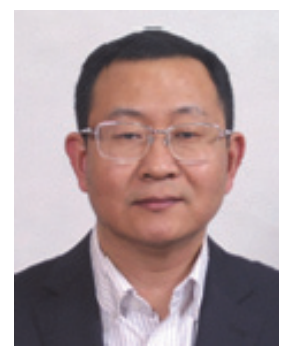

黎占亭, 1966 年出生于河南, 1985 年郑州大学化学系毕业, 1992 年在中科院上海有机化学研究所获博士学位(导师: 陈庆 云研究员), 先后在南丹麦大学和美国伊利诺伊大学做博士后 研究, 1993 2010 年任上海有机所副研究员和研究员, 现任复 旦大学化学系教授. 主要从事物理有机、超分子化学和有机孔 材料研究.

\section{References}

[1] Whitesides, G. M.; Mathias, J. P.; Seto, C. T. Science 1991, 254, 1312.

[2] Jiang, B.; Guo, D.; Liu, Y. Prod. Chem. 2013, 25, 869. (蒋邦平, 郭 东升, 刘育, 化学进展, 2013, 25, 869.)

[3] Yi, J.; Xiao, X.; Zhang, Y.; Xue, S.; Tao, Z.; Zhang, J. Acta Chim. Sinica 2014, 72, 949. (易君明, 肖欣, 张云黔, 薛赛凤, 陶朱, 张 建新, 化学学报, 2014, 72, 949.)

[4] Zou, Q.; Zhang, J.; Tian, H. Prod. Chem. 2012, 24, 1632. (邹祺, 张 隽佶, 田禾, 化学进展, 2012, 24, 1632.)

[5] Zhou, L.; Xu, L.; Gong, H. Acta Chim. Sinica 2014, 72, 447. (周丽, 徐立进, 龚汉元, 化学学报, 2014, 72, 447.)

[6] Li, C.; Zhao, X.; Gao, X.; Wang, Q.; Li, Z. Chin. J. Chem. 2013, 31, 582.
[7] Pan, G.; Feng, Z.; Wei, J.; Yu, Y. Acta Chim. Sinica 2013, 71, 733. (潘高翔, 冯泽, 韦嘉, 俞燕蕾, 2013, 71, 733)

[8] Shi, Z.; Song, Y.; Lu, F.; Zhou, T.; Zhao, X.; Zhang, W.; Li, Z. Acta Chim. Sinica 2013，71，51. (施朱明，宋宇，陆方，周天佑，赵新, 张文科, 黎占亭, 化学学报, 2013, 71, 51.)

[9] Wan, Q. T.; Li, Z. T. Imag. Sci. Photochem. 2015, 33, 3. (万堂庆, 黎占亭, 影像科学与光化学, 2015, 33, 3.)

[10] Tung, C.-H.; Wu, L.-Z.; Zhang, L.-P.; Chen, B. Acc. Chem. Res. 2003, 36, 39 .

[11] Wang, M.-X. Chem. Commun. 2008, 4541.

[12] Jiang, X.; Feng, M.; Zhang, D.; Wang, B.; Dong, Z.; Gao, G. Chin. J. Chem. 2013, 31, 673 .

[13] Chen, Y.; Liu, Y. Chem. Soc. Rev. 2010, 39, 495.

[14] Chen, C.-F. Chem. Commun. 2011, 47, 1674.

[15] Zhang, M.; Zhu, K.; Huang, F. Chem. Commun. 2010, 46, 8131.

[16] Duan, P.; Cao, H.; Zhang, L.; Liu, M. Soft Matter. 2014, 10, 5428.

[17] Li, H.; Yang, Y.-W. Chin. Chem. Lett. 2013, 24, 545.

[18] Wang, Y.; Xu, H.; Zhang, X. Adv. Mater. 2009, 21, 2849.

[19] Zhao, Y.; Sakai, F.; Su, L.; Liu, Y.; Wei, K.; Chen, G.; Jiang, M. Adv. Mater. 2013, 25, 5215 .

[20] Yi, J.-M.; Ni, X.-L.; Xiao, X.; Lu, L.-B.; Xue, S.-F.; Zhu, Q.-J.; Tao, Z. Chin. Chem. Lett. 2013, 24, 362.

[21] Li, S.-L.; Xiao, T.; Lin, C.; Wang, L. Chem. Soc. Rev. 2012, 41, 5950.

[22] Du, M.; Bu, X. Prog. Chem. 2009, 21, 2458. (杜沝，卜显和, 化学 进展, 2009, 21, 2458.)

[23] Zeng, M.-H.; Feng, X.-L.; Zhang, W.-X.; Chen, X.-M. Dalton Trans. 2006, 5294.

[24] Zhuang, C.-F.; Zhang, J.; Wang, Q.; Chu, Z.-H.; Fenske, D.; Su, C.-Y. Chem. Eur. J. 2009, 15, 7578.

[25] Jia, J.; Wang, L.; Zhao, Q.; Sun, F.; Zhu, G. Acta Chim. Sinica 2013 71, 1492. (䍙江涛, 王蕾, 赵晴, 孙福兴, 朱广山, 化学学报, 2013, $71,1492$.

[26] Zhu, L.; Zhang, D.; Qiu, S. Sci. China, Chem. 2014, 44, 229. (朱良 奎, 张大梁, 装式纶, 中国科学: 化学, 2014, 44, 229.)

[27] Xuan, W.; Zhu, C.; Liu, Y.; Cui, Y. Chem. Soc. Rev. 2012, 41, 1677.

[28] Cui, Y.; Chen, B.; Qian, G. Coord. Chem. Rev. 2014, 273 274, 76.

[29] Sun, C.-Y.; Qin, C.; Wang, X.-L.; Su, Z.-M. Expert Opin. Drug Deliv. 2013, 10, 89 .

[30] Tian, J.; Xu, J.; Zhu, F.; Lu, T.; Su, C.; Ouyang, G. J. Chromatogr. A 2013, 1300, 2.

[31] Ding, S.-Y.; Gao, J.; Wang, Q.; Zhang, Y.; Song, W.-G.; Su, C.-Y.; Wang, W. J. Am. Chem. Soc. 2011, 133, 19816.

[32] Zhou, T.-Y.; Xu, S.-Q.; Wen, Q.; Pang, Z.-F.; Zhao, X. J. Am. Chem. Soc. 2014, 136, 15885

[33] Ding, H.; Li, Y.; Hu, H.; Sun, Y.; Wang, J.; Wang, C.; Wang, C.; Zhang, G.; Wang, B.; Xu, W.; Zhang, D. Chem. Eur. J. 2014, 20, 14614.

[34] Wang, P.; Kang, M.; Sun, S.; Liu, Q.; Zhang, Z.; Fang, S. Chin. J. Chem. 2014, 32, 838 .

[35] Liu, X.-H.; Guan, C.-Z.; Ding, S.-Y.; Wang, W.; Yan, H.-J.; Wang, D.; Wan, L.-J. J. Am. Chem. Soc. 2013, 135, 10470.

[36] Zhang, X.; Zeng, Q.; Wang, C. Nanoscale 2013, 5, 8269.

[37] Huang, F.; Zhai, C.; Zheng, B.; Li, S. Supramolecular Polymers, Zhejiang University Press, Hangzhou, 2012, pp. 1 175. (黄飞鹤, 翟春熙，郑波，李世军，超分子聚合物，浙江大学出版社，杭州, 2012, pp. $1 \sim 175$ ).

[38] Kong, R.; Shi, D.; Liu, R.; Wu, C.; Ni, P.; Chen, M. Acta Chim. Sinica 2013, 71, 1540. (孔芯, 施冬健, 刘蓉瑾, 吴超, 倪沛红, 陈 明清, 化学学报, 2013, 71, 1540.)

[39] Han, Y.; Meng, Z.; Ma, Y.-X.; Chen, C.-F. Acc. Chem. Res. 2014, 47, 2026.

[40] Zhao, Y.-C.; Zhang, L.-M.; Wang, T.; Han, B.-H. Polym. Chem. 2014, 5, 614 .

[41] Berl, V.; Schmutz, M.; Krische, M. J.; Khoury, R. G.; Lehn, J. M. Chem. Eur. J. 2002, 8, 1227.

[42] Lightfoot, M. P.; Mair, F. S.; Pritchard, R. G.; Warren, J. E. Chem. Commun. 1999, 1945.

[43] Palmans, A. R. A.; Meijer, E. W. Angew. Chem., Int. Ed. 2007, 46, 8948.

[44] Fang, R.; Liu, Y.; Wang, Z.; Zhang, X. Polym. Chem. 2013, 4, 900.

[45] Zhang, Q.; Qu, D. H.; Ma, X.; Tian, H. Chem Commun. 2013, 49, 9800.

[46] Li, C.; Han, K.; Li, J.; Zhang, Y.; Chen, W.; Yu, Y.; Jia, X. Chem. Eur. J. 2013, 19, 11892 
[47] Hirao, T.; Tosaka, M.; Yamago, S.; Haino, T. Chem. Eur. J. 2014, $20,16138$.

[48] Zhang, K.-D.; Tian, J.; Hanifi, D.; Zhang, Y.; Sue, A. C. H.; Zhou, T.-Y.; Zhang, L.; Zhao, X.; Liu, Y.; Li, Z.-T. J. Am. Chem. Soc. 2013, 135, 17913.

[49] Zhang, Z.-J.; Zhang, Y.- M.; Liu, Y. J. Org. Chem. 2011, 76, 4682.

[50] Zhang, Y.; Zhou, T.-Y.; Zhang, K.- D.; Dai, J.-L.; Zhu, Y.-Y.; Zhao, X. Chem. Asian J. 2014, 9, 1530.

[51] Zhang, L.; Zhou, T.-Y.; Tian, Y.; Wang, H.; Zhang, D.-W.; Zhao, X.; Liu, Y.; Li, Z.-T. Polym. Chem. 2014, 5, 4715.

[52] Zhang, D.-W.; Tian, J.; Chen, L.; Zhang, L.; Li, Z.-T. Chem. Asian J. 2015, 10, 56.

[53] Singh, A.; Tolev, M.; Meng, M.; Klenin, K.; Plietzsch, O.; Schilling,
C. I.; Muller. T.; Nieger, M.; Bräse, S.; Wenzel, W.; Richert, C. Angew. Chem. Int. Ed. 2011, 50, 3227.

[54] Liu, Y.; Huang, Z.; Liu, K.; Kelgtermans, H.; Dehaen, W.; Wang, Z.; Zhang, X. Polym. Chem. 2014, 5, 53

[55] Zhou, C.; Tian, J.; Wang, J.-L.; Zhang, D.-W.; Zhao, X.; Liu, Y.; Li, Z.-T. Polym. Chem. 2014, 5, 341.

[56] Tian, J.; Ding, Y.-D.; Zhou, T.-Y.; Zhang, K.-D.; Zhao, X.; Wang, H.; Zhang, D.-W.; Liu, Y.; Li, Z.-T. Chem. Eur. J. 2014, 20, 575.

[57] Chen, L.; Zhang, S.-C.; Wang, H.; Zhou, Y.-M.; Li, Z.-T.; Zhang, D.-W. Tetrahedron 2014, 70, 4778.

[58] Tian, J.; Zhou, T.-Y.; Zhang, S.-C.; Aloni, S.; Altoe, M. V.; Xie, S.-H.; Wang, H.; Zhang, D.-W.; Zhao, X.; Liu, Y.; Li, Z.-T. Nat. Commun. 2014, 5, 5574 .

(Zhao, C.) 